\title{
MATHEMATICAL INEQUALITIES FOR BIPARAMETRIC EXTENDED INFORMATION MEASURES
}

\author{
Flavia-Corina Mitroi And Nicuşor Minculete
}

Abstract. In this paper we introduce quasilinear-type divergences defined by the two-parameter generalization of the logarithm. Jeffreys and Jensen-Shannon divergence are also extended to biparametric forms.

Mathematics subject classification (2010): 26D15, 94A17.

Keywords and phrases: Tsallis relative entropy, Jeffreys divergence, Jensen-Shannon divergence, quasilinear divergence.

\section{REFERENCES}

[1] J. M. AldAZ, A stability version of Hölder's inequality, J. Math. Anal. Appl. 343, 2 (2008), 842-852.

[2] S. ARIMOTO, Information-theoretic considerations on estimation problems, Information and Control 19 (1971), 181-190.

[3] E. Boekee And J. C. A. Van Der Lubbe, The R-norm Information Measure, Information and Control 45 (1980), 136-155.

[4] S. S. DRAGOMIR, J. ŠUndE AND C. BuşE, New inequalities for Jeffreys divergence measure, Tamsui Oxf. J. Math. Sci. 16 (2000), 295-309.

[5] S. S. DRAGOMiR, Bounds for the normalized Jensen functional, Bull. Austral. Math. Soc. 74, 3 (2006), 417-478.

[6] A. Ibrahim, S. S. Dragomir, M. Darus, Power series inequalities related to Young's inequality and applications, Online: http://rgmia.org/papers/v15/v15a52.pdf

[7] S. Furuichi, On refined Young inequalities and reverse inequalities, J. Math. Ineq. 5 (2011), 21-31.

[8] S. Furuichi, N. Minculete And F.-C. Mitroi, Some inequalities on generalized entropies, arXiv:1104.0360v1. To appear at J. Inequal. Appl.

[9] S. Furuichi, F.-C. Mitroi, Mathematical inequalities for some divergences, Physica A 391 (2012), 388-400. DOI:10.1016/j . physa.2011.07.052

[10] H. JefFreys, An invariant form for the prior probability in estimation problems, Proc. Roy. Soc. Lon., Ser. A 186 (1946), 453-461.

[11] V. SCHWÄMMLE AND C. TSALLIS, Two-parameter generalization of the logarithm and exponential functions and Boltzmann-Gibbs-Shannon entropy, J. Math. Phys. 48 (2007), 113301.

[12] C. Ts allis, Possible generalization of Boltzmann-Gibbs statistics, J.Stat. Phys. 52 (1988), 479-487. 